\title{
A prefrontal non-opioid mechanism in placebo analgesia
}

\author{
Predrag Petrovic ${ }^{\mathrm{a}, *}$, Eija Kalso ${ }^{\mathrm{b}}$, Karl Magnus Petersson ${ }^{\mathrm{a}, \mathrm{c}}$, Jesper Andersson ${ }^{\mathrm{d}}$, Peter Fransson ${ }^{\mathrm{a}}$, \\ Martin Ingvar ${ }^{\mathrm{a}}$ \\ a Cognitive Neurophysiology Research Group, Stockholm Brain Institute, Osher Center for Integrative Medicine, Karolinska Institutet, Stockholm, Sweden \\ ${ }^{\mathrm{b}}$ Pain Clinic, Department of Anaesthesiology and Intensive Care Medicine, Helsinki University Central Hospital and Institute of Clinical Medicine, University of Helsinki, Finland \\ ${ }^{\mathrm{c}}$ Cognitive Neuroscience Research Group, IBB/CBME, University of Algarve, Faro, Portugal \\ ${ }^{\mathrm{d}}$ Oxford Centre for Functional MRI of the Brain, University of Oxford, Oxford, UK
}

\section{A R T I C L E I N F O}

\section{Article history:}

Received 23 August 2008

Received in revised form 17 February 2010

Accepted 10 March 2010

\section{Keywords:}

Placebo

Pain

Opioid

Cognitive

Orbitofrontal cortex, prefrontal cortex,

anterior cingulate cortex

PET

fMRI, expectation, error signal

\begin{abstract}
A B S T R A C T
Behavioral studies have suggested that placebo analgesia is partly mediated by the endogenous opioid system. Expanding on these results we have shown that the opioid-receptor-rich rostral anterior cingulate cortex (rACC) is activated in both placebo and opioid analgesia. However, there are also differences between the two treatments. While opioids have direct pharmacological effects, acting on the descending pain inhibitory system, placebo analgesia depends on neocortical top-down mechanisms. An important difference may be that expectations are met to a lesser extent in placebo treatment as compared with a specific treatment, yielding a larger error signal. As these processes previously have been shown to influence other types of perceptual experiences, we hypothesized that they also may drive placebo analgesia. Imaging studies suggest that lateral orbitofrontal cortex (lObfc) and ventrolateral prefrontal cortex (vlPFC) are involved in processing expectation and error signals. We re-analyzed two independent functional imaging experiments related to placebo analgesia and emotional placebo to probe for a differential processing in these regions during placebo treatment vs. opioid treatment and to test if this activity is associated with the placebo response. In the first dataset lObfc and vlPFC showed an enhanced activation in placebo analgesia vs. opioid analgesia. Furthermore, the rACC activity co-varied with the prefrontal regions in the placebo condition specifically. A similar correlation between rACC and vlPFC was reproduced in another dataset involving emotional placebo and correlated with the degree of the placebo effect. Our results thus support that placebo is different from specific treatment with a prefrontal top-down influence on rACC.

(C) 2010 International Association for the Study of Pain. Published by Elsevier B.V. All rights reserved.
\end{abstract}

\section{Introduction}

It is widely accepted that placebo analgesia is mediated by the endogenous opioid system since the opioid receptor antagonist naloxone attenuates the placebo effect $[2,5-7,26]$. In line with this we have shown a similar functional anatomy of opioid and placebo analgesia [34]. Opioid-rich regions including the rostral anterior cingulate cortex (rACC) were involved in both conditions. Our initial findings have been replicated and extended $[8,21,51,52,58]$. In a recent functional MRI (fMRI) study, we generalized our previous results on placebo analgesia [34] to emotional processing which showed a similar pattern of placebo-dependent activation [36].

Opioid analgesia is mediated through a direct pharmacological activation of the endogenous opioid receptors and shares the prop-

\footnotetext{
* Corresponding author at: MR Research Center N8, Department of Clinical Neuroscience, Karolinska Institute, 17176 Stockholm, Sweden. Tel.: +46 735101211; fax: +468344146.

E-mail addresses: predrag.petrovic@ki.se, predrag.petrovic@cns.ki.se (P. Petrovic).
}

erties of a placebo manipulation initially. However, differences in the cerebral processing would be expected during ongoing treatment especially in terms of expectation and error signals - processes that have shown to profoundly modulate visual perception and emotional experiences $[17,28,44,57]$. In the opioid condition, expectations for effective analgesia are met and therefore remain congruent with the level of nociceptive processing. On the other hand, in the placebo condition, the treatment expectation is upheld by means of the placebo manipulation that is incongruent with processing level of the nociceptive input. Potentially, this would result in an error signal between the expectations and the nociceptive processing. The expression of such an error signal in pain processing has been demonstrated by means of deliberate manipulations of the expected intensity of pain [43] where the error signal correlated with an increased activity in the lateral orbitofrontal cortices. An important question is whether expectations and error processing directly relate to placebo analgesia as has been indicated in other perceptual experiences and emotion [17,28,44,57]. Behavioral results indicate such a relationship as the level of explicit expectation has been shown to correlate to the subsequently reported pain level [39]. 
Orbitofrontal- and ventrolateral prefrontal cortex have been suggested to be involved in higher order emotional and pain regulations [32,35] including placebo analgesia [21,27,34,51], and error and expectation processings $[18,31,43]$. However, these regions do not show a clear opioid receptor binding or opioid-induced activation in functional imaging studies $[10,15,19,34,53,55,56]$. In the present study we hypothesized that the prefrontal regions code for the expected nociceptive processing and error signals, which then influence the descending opioid system in the ACC specifically during placebo analgesia. In that case, the prefrontal activity and the interaction with the ACC should be more pronounced in the placebo condition than in the opioid condition. Moreover, the functional relation between the prefrontal regions and ACC should correlate with the reported placebo effect. To test this suggestion we re-analyzed the dataset from our previous placebo analgesia PET study [34] and emotional placebo fMRI study [36].

\section{Methods}

The data presented here represent a re-analysis of the two previously presented materials $[34,36]$. The re-analysis involves new contrast and functional connectivity analyses.

\subsection{Study 1 - PET study on placebo and opioid analgesia}

The first study focused on placebo and opioid analgesia [34]. In this positron emission tomography (PET) study on the placebo analgesia effect nine healthy male subjects participated (age: 2027 years). Most of the results have been presented in a previous article [34].

In the present analysis two conditions were further compared: (1) pain and opioid treatment, i.e. opioid analgesia (POP) and (2) pain and placebo treatment, i.e. placebo analgesia (PPL). In both conditions the subjects had been stimulated with a tonic noxious heat stimulation $\left(48^{\circ} \mathrm{C}, 70 \mathrm{~s}\right.$, stimulation area $\left.=2 \times 3 \mathrm{~cm}\right)$ on the dorsum of the left hand. In the POP condition the subjects had been pre-treated with a short-lasting opioid (remifentanil $0.5 \mu \mathrm{g} / \mathrm{kg}$ ) intravenously. In the PPL condition the subjects had been given saline intravenously but they assumed that a strong analgesic was administered. Both treatments were given in a double blind fashion. Both conditions had been performed twice in each subject. For more detailed methods see [34]. For completeness we also modeled the other original conditions in the present re-analysis (Pain stimulation only - P; Warm stimulation and opioid treatment - WOP; Warm stimulation and placebo treatment - WPL; Warm stimulation only - W). However, since these conditions are not a part of the present analysis we will not discuss them further.

In the present re-analysis we used SPM99 (http://www.fil.ion.ucl.ac.uk/spm) for pre-processing and statistical analysis of the data. A fixed-effect approach was used for the PET analysis as in the original analysis, i.e. each data point represents a measurement (two for each subject). All conditions (POP, PPL, P, WOP, WPL and W) were entered in the model as in the original analysis. We chose not to re-analyze the dataset in a random effect model since the power was too low due to the limited sample size in the original PET study. The result has therefore an inference only on a group level (i.e. the results are valid for the group of individuals that took part in the study) and not on a population level. A subtraction analysis was performed to test for differences between the PPL and the POP conditions, i.e. (PPL-POP) and (POP-PPL). For the contrast PPL-POP an exclusive masking procedure was implemented so that all activations induced by general decreases in the opioid condition vs. the pain condition (i.e. P-POP) with a threshold of $P$ uncorrected $=0.05$ were removed. This procedure indicates that the remaining findings in PPL-POP could be attributed to increased activity in the PPL condition and not decreased activity in the POP condition. A threshold of $P$-value (uncorrected) $<0.001$ was set for reporting results in $\mathrm{rACC}$ and vlPFC/Obfc since our hypothesis involved specifically these regions. We also report when the activity is bordering another region such as anterior insula.

In the present re-analysis we also performed a functional connectivity analysis between the $\mathrm{rACC}$ and the vlPFC/lObfc in the placebo (PPL) and the opioid (POP) conditions. In the voxel showing the highest $Z$-value in the rACC during the main effect of opioids $[X Y Z]=\left[\begin{array}{lll}8 & 44 & 12\end{array}\right]$ the adjusted activity was extracted from all conditions (Fig. 1). The adjusted activity for each scan was used as a covariate of interest for each condition in a general linear model and the conditions were modeled as confounding covariates. We then studied the regressions between the rACC and the vlPFC/lObfc during the placebo (PPL) and the opioid (POP) conditions. In addition, differences in the observed regressions between the conditions were studied by employing a simple contrast analysis in SPM99.

\subsection{Study 2 - fMRI study on emotional placebo}

In the second study, the placebo effect was probed during emotional processing by giving a non-specific treatment that subjects believed was an effective anxiolytic substance before presentation of unpleasant pictures [36]. In this functional magnetic resonance imaging (fMRI) study on emotional placebo effect 15 healthy female subjects participated (age: 20-33). On day 1 (expectation induction) the subjects watched three blocks of unpleasant and neutral pictures (13 unpleasant and 13 neutral pictures; stimulus duration was $4 \mathrm{~s}$; inter-stimulus interval was $7 \mathrm{~s}$ ). The experience of unpleasantness was lowered after the subjects received a small intravenous dose of anxiolytic (midazolam $0.015 \mathrm{mg} / \mathrm{kg}$ ) but reversed after they received a blocker of the anxiolytic drug (flumazenil $0.25 \mathrm{mg}$ ). This way we induced treatment expectations that the anxiolytic drug was highly effective in reducing unpleasantness. On day 2 (placebo testing) the subjects were scanned in a 1.5-T scanner while they watched similar pictures (each block contained 10 unpleasant and 10 neutral pictures; stimulus duration was $4 \mathrm{~s}$; inter-stimulus interval was jittered but $12.2 \mathrm{~s}$ in average). In some blocks the subjects thought that they had received the anxiolytic drug (but had actually only received saline intravenously) and rated the negative pictures as less unpleasant than when they believed that they had received the anxiolytic blocker. Thus, in some blocks a placebo effect had been accomplished

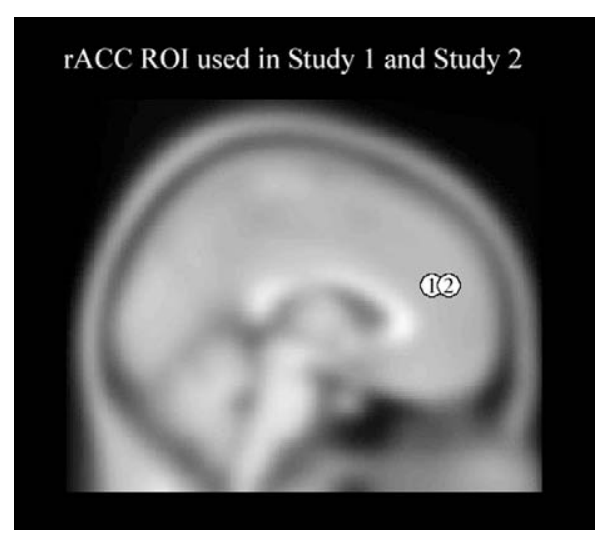

Fig. 1. The ROIs in the rACC used for the functional connectivity analysis are shown in the SPM-template below. In Study 1 the ROI was focused on the region showing the most expressed activations in the rACC after opioid treatment $\left([X Y Z]=\left[\begin{array}{lll}8 & 44 & 12\end{array}\right]\right)$. In Study 2 the ROI was focused on the most expressed placebo-induced activation in the placebo responders after controlling for unspecific placebo effects $\left([X Y Z]=\left[\begin{array}{lll}-12 & 48 & 12\end{array}\right]\right)$. 
(placebo condition) while the other blocks were used as a baseline (control condition). Taking the different visual stimulations (unpleasant and neutral pictures) into account, there were also four event-related conditions in total: unpleasant pictures and placebo (UP), neutral pictures and placebo (NP), unpleasant pictures and control treatment (UC), and neutral pictures and control treatment (NC). Each subject went through three sessions, each one consisting of one placebo block and one control block. For further details see [36]. After each block of pictures the experience of unpleasantness was measured using a visual analogue scale (VAS) ranging from 0 (no unpleasantness) to 100 (maximal imagined unpleasantness). The behavioral placebo response was measured by subtracting the unpleasantness rating during the control condition with the unpleasantness rating during the placebo condition.

In the present analysis we used data from the study on emotional placebo described above [36] to try (1) to reproduce the findings of a placebo-specific functional connectivity between rACC and the vlPFC/lObfc for each subject and (2) to investigate whether this functional relation relates to the degree of placebo effect (this analysis was not possible for the PET study because the functional connectivity could only be performed on a group level). The activity of the rACC was represented by a region of interest (ROI) with a radius of $3 \mathrm{~mm}$, centered on the voxel showing the highest $Z$-score in rACC for the placebo responders in the study of emotional placebo $([X Y Z]=[-124812])$ (Fig. 1; [36]).

Firstly, we performed within-subject analysis. For each subject, the activity, adjusted for session effects, was extracted for these voxels in the placebo and the control blocks. The average activity for each condition was subtracted from the adjusted activity for each scan (mean correction). These activity values were then used as condition-specific covariates of interest in a GLM implemented in SPM99 (http://www.fil.ion.ucl.ac.uk/spm) to generate regressions between the rACC-ROI and the rest of the brain during placebo condition (rPC) and control condition (rCC), in which the two treatment conditions (placebo and control conditions) and the four event-related conditions (UP, NP, UC and NC) were modeled as confounding covariates. The functional connectivity effect we were interested in this analysis was the regression between rACC-ROI and orbitofrontal-/ventrolateral prefrontal cortex in both the placebo condition (rPC) and the control condition (rCC). We also studied the differences between the regressions in placebo condition and control condition (rPC-rCC). The analysis was performed for each subject, however, in two of the subject spikes were detected in one of the three sessions, which therefore had to be removed.

Secondly, we performed a group analysis. In order to test whether the functional relation between rACC and prefrontal cortex in each subject (see above) was related to the degree of placebo effect, a second-level random effect analysis was performed. In this analysis the functional connectivity result for each subject was correlated with the reported placebo effect. This analysis will show whether the functional connectivity between the rACC and the vIPFC is most pronounced in the best placebo responders. Since we were only interested in the functional relation between $\mathrm{rACC}$ and vlPFC/lObfc a threshold of $P$-uncorrected $<0.001$ was set for reporting results in vlPFC and lObfc. Since one of the subjects' behavioral data were lost only 14 subjects were enrolled in the second-level analysis.

\section{Results}

\subsection{Results from Study 1 - PET study on placebo and opioid analgesia}

The results directly comparing the condition involving pain and opioid treatment (i.e. opioid analgesia; POP) with pain and placebo treatment (i.e. placebo analgesia; PPL) are shown below. Here, we tested whether ACC was more involved in opioid treatment condition, while the prefrontal areas (lObfc and vlPFC) were more involved in the placebo condition. Moreover, we tested whether the functional relation between rACC and the prefrontal regions was more expressed in the placebo condition than in the opioid condition. Analyses assessing the general placebo effect are presented elsewhere [34].

\subsubsection{Opioid effect vs. placebo effect in pain (POP-PPL; Table 1)}

The rACC (including the subgenual ACC) was significantly more activated in the opioid condition as compared with the placebo condition. Likewise a region overlapping anterior insula and lObfc was bilaterally more activated in this contrast. Upon inspection this activation only marginally stretched into lObfc.

\subsubsection{Placebo effect vs. opioid effect in pain (PPL-POP; Table 2)}

The lObfc was significantly more activated in the placebo condition as compared with the opioid condition (Fig. 2A). This activation was most pronounced in the right hemisphere in terms of both the number of activated voxels and significance scores (in the left lObfc this activation did not reach significance). However, no formal comparison was made in the present dataset. The right lObfc activation stretched up into the right vlPFC in a continuum of activated voxels (Fig. 2B). The placebo-dependent activations survived the exclusive masking indicating that they were true activations in the placebo condition rather than decreases in the opioid condition.

\subsubsection{Functional connectivity analysis (Table 3)}

In this analysis the activity in rACC was co-varied with the rest of the brain in order to characterize the connectivity between rACC and PFC (see Section 2). rACC showed a significant co-variation with right and left lObfc and right vlPFC in the PPL condition. No such effect was shown in the POP condition. When these two regressors were compared it was shown that the rACC co-varied significantly more with the right vlPFC and lObfc in the placebo condition than in the opioid condition (Fig. 2C and D).

\subsection{Results from Study 2 - fMRI study of emotional placebo}

A regression analysis was performed on the dataset involving emotional placebo [36] in an attempt to reproduce the results mentioned above indicating a placebo-specific functional relation between rACC and VIPFC, and to test whether this connectivity correlated with the placebo response (see Section 2). This study has previously shown a placebo-specific increase in rACC and several regions of $1 \mathrm{Obfc} / \mathrm{vlPFC}$ in the subtraction analysis (see further [36]). The placebo response-related activity (UP-UC) in prefrontal cortex also correlated with the behavioral placebo response in the vlPFC (but not in the lObfc): [32 46 8] $t=5.89$ (Fig. 3A and B; [36]).

Table 1

Regions activated more by opioid (remifentanil $0.5 \mu \mathrm{g} / \mathrm{kg}$ ) than by placebo during pain (Study 1). In the present analysis a fixed effect design was used.

\begin{tabular}{lrrrrrr} 
Region & \multicolumn{3}{c}{ MNI coordinates } & & \multirow{2}{*}{ t-value } & P-value \\
\cline { 2 - 4 } & $X$ & $Y$ & $Z$ & & \\
\cline { 1 - 4 } Opioid analgesia vs. placebo & analgesia (POP-PPL) & & \\
rACC (BA 24) & 6 & 52 & 10 & 4.95 & $<0.001$ \\
Subgenual ACC/mObfc & 0 & 22 & -14 & 3.48 & $<0.001$ \\
Right anterior insula/lObfc & 50 & 10 & 2 & 4.80 & $<0.001$ \\
Left anterior insula/lObfc & -46 & 4 & 2 & 5.23 & $<0.001$ \\
\hline
\end{tabular}

(r)ACC, (rostral) anterior cingulate cortex; mObfc, medial orbitofrontal cortex; lObfc, lateral orbitofrontal cortex. 


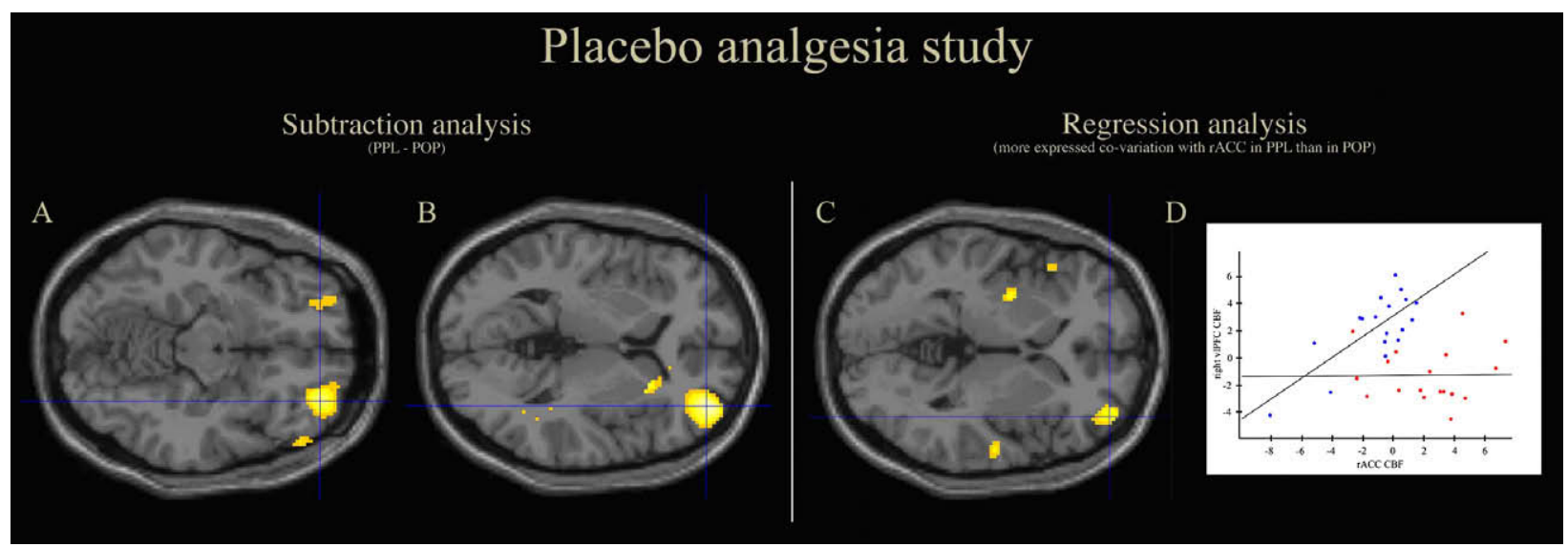

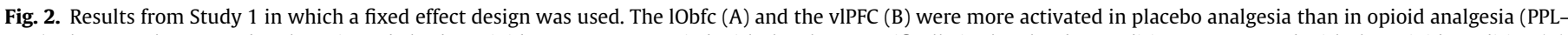

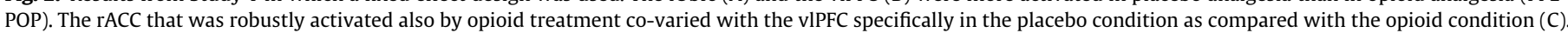
Adjusted rCBF activity indicates this relation for placebo (blue dots) but not for opioid (red dots) treatment (D).

\subsection{1. rACC co-variation (Table 4)}

Ten of the 15 subjects showed a placebo-specific co-variation between the rACC and the vlPFC/lObfc (see Supplementary Table 1). In the second-order random effect analysis we probed whether this functional relation correlated with the behavioral placebo effect. The co-variation between $\mathrm{rACC}$ and vlPFC/lObfc tended to be most expressed for the best placebo responders. No such effects were observed in the control condition, and the co-variation between rACC and vlPFC was shown to be significantly stronger in the placebo condition than in the control condition (Fig. 3C and D).

\section{Discussion}

In the present study we extended the analysis of two previous placebo studies in order to characterize a cognitive non-opioid component of the placebo response. In the re-analysis of the PET study on placebo analgesia [34] we observed that orbitofrontal cortex and vlPFC were significantly more activated during placebo treatment than opioid treatment. Moreover, rACC displayed a functional connectivity with these prefrontal regions specifically during the placebo context. In the re-analysis of the fMRI study on emotional placebo [36] we showed that the functional relation between rACC and vlPFC was associated with the degree of placebo response.

In our original placebo analgesia study [34] we showed a neurophysiological correlate to previous behavioral studies indicating a relation between the endogenous opioid system and placebo analgesia $[2,5-7,26]$. The study showed a co-activated network in opioid and placebo treatments including the rACC and the anterior insula stretching into lObfc. Several other functional imaging studies have also indicated an involvement of rACC in the placebo response [8,21,51,52,58]. Auto-radiographic and post-mortem studies on humans, primates and rats $[3,38,54]$, opioid receptor

Table 2

Regions activated more by placebo than by opioid (remifentanil $0.5 \mu \mathrm{g} / \mathrm{kg}$ ) during pain (Study 1). In the present analysis a fixed effect design was used.

\begin{tabular}{|c|c|c|c|c|c|}
\hline \multirow[t]{2}{*}{ Region } & \multicolumn{3}{|c|}{ MNI coordinates } & \multirow[t]{2}{*}{$t$-value } & \multirow[t]{2}{*}{$P$-value } \\
\hline & $X$ & Y & $Z$ & & \\
\hline \multicolumn{6}{|c|}{ Placebo analgesia vs. opioid analgesia (PPL-POP) } \\
\hline Right vlPFC $*$ & 36 & 52 & 4 & 4.43 & $<0.001$ \\
\hline Right $10 b c^{*}$ & 32 & 50 & -12 & 4.04 & $<0.001$ \\
\hline Left lobfc ${ }^{*}$ & -24 & 52 & -18 & 3.07 & ns \\
\hline
\end{tabular}

lObfc, lateral orbitofrontal cortex; vlPFC, ventrolateral prefrontal cortex; *, indicates regions surviving the exclusive masking procedure. binding studies $[19,55,56]$, and blood flow studies $[1,10,15,53]$ on human subjects indicate that ACC and insula have high concentrations of opioid receptors. Altogether, these studies suggest that the cortical opioid system is involved in mediating placebo analgesia. Indeed, recent opioid receptor binding PET-studies have shown an opioid response in the rACC during placebo analgesia [52,58].

Here, we show that opioid-rich areas in rACC and the anterior insula were more extensively activated during opioid as compared with placebo analgesia in line with the stronger analgesic effect in the opioid condition. The activation of these areas was most likely due to direct activation of opioid receptors by the exogenous opioid (remifentanil). Conversely, the response was opposite in the IObfc and the vlPFC, that is, a significantly larger activation was observed for placebo analgesia than opioid analgesia (Fig. 2A and B). Raw data suggest that the binding potential in these prefrontal regions is not as high as in ACC and insula $[19,55,56]$ and they have not shown increased activation by opioids in other imaging studies $[1,10,15,53]$. The previously presented small opioid-induced lObfc activation [34] was only observed on the border of an extensive insula activation. In fact, the activation observed in lObfc may represent a smoothing effect of the data. Hence, although it has not been shown whether lObfc/vlPFC has a lower concentration of opioid receptors than ACC and insula, the prefrontal regions seem to be involved in an opioid response to a lesser degree and may be more important for other cognitive processes.

Table 3

Analysis of the functional connectivity between rACC and vlPFC/lObfc in the placebo and opioid conditions (Study 1). In the present analysis a fixed effect design was used.

\begin{tabular}{|c|c|c|c|c|c|}
\hline \multirow{2}{*}{$\begin{array}{l}\text { Region co-varying with rACC } \\
{[X Y Z]=\left[\begin{array}{lll}8 & 44 & 12\end{array}\right]}\end{array}$} & \multicolumn{3}{|c|}{ MNI coordinates } & \multirow[t]{2}{*}{$t$-value } & \multirow[t]{2}{*}{$P$-value } \\
\hline & $X$ & $Y$ & $Z$ & & \\
\hline \multicolumn{6}{|l|}{ Placebo analgesia (PPL) } \\
\hline Right lObfc/anterior insula & 34 & 18 & -18 & 4.05 & $<0.001$ \\
\hline Right lObfc & 42 & 36 & -4 & 3.45 & $<0.001$ \\
\hline Right vlPFC & 36 & 52 & 6 & 3.90 & $<0.001$ \\
\hline Left $10 b f c / a n t e r i o r$ insula & -24 & 14 & -18 & 4.45 & $<0.001$ \\
\hline Left lObfc & -28 & 36 & -14 & 4.40 & $<0.001$ \\
\hline \multicolumn{6}{|l|}{ Opioid analgesia (POP) } \\
\hline- & - & - & - & - & - \\
\hline \multicolumn{6}{|c|}{ Placebo analgesia vs. opioid analgesia (PPL-POP) } \\
\hline Right vlPFC & 40 & 54 & 0 & 3.16 & 0.001 \\
\hline Right lObfc & 24 & 18 & -24 & 3.38 & $<0.001$ \\
\hline Left lObfc & -26 & 42 & -20 & 3.60 & $<0.001$ \\
\hline
\end{tabular}

rACC, rostral anterior cingulate cortex; lObfc, lateral orbitofrontal cortex; vlPFC, ventrolateral prefrontal cortex. 


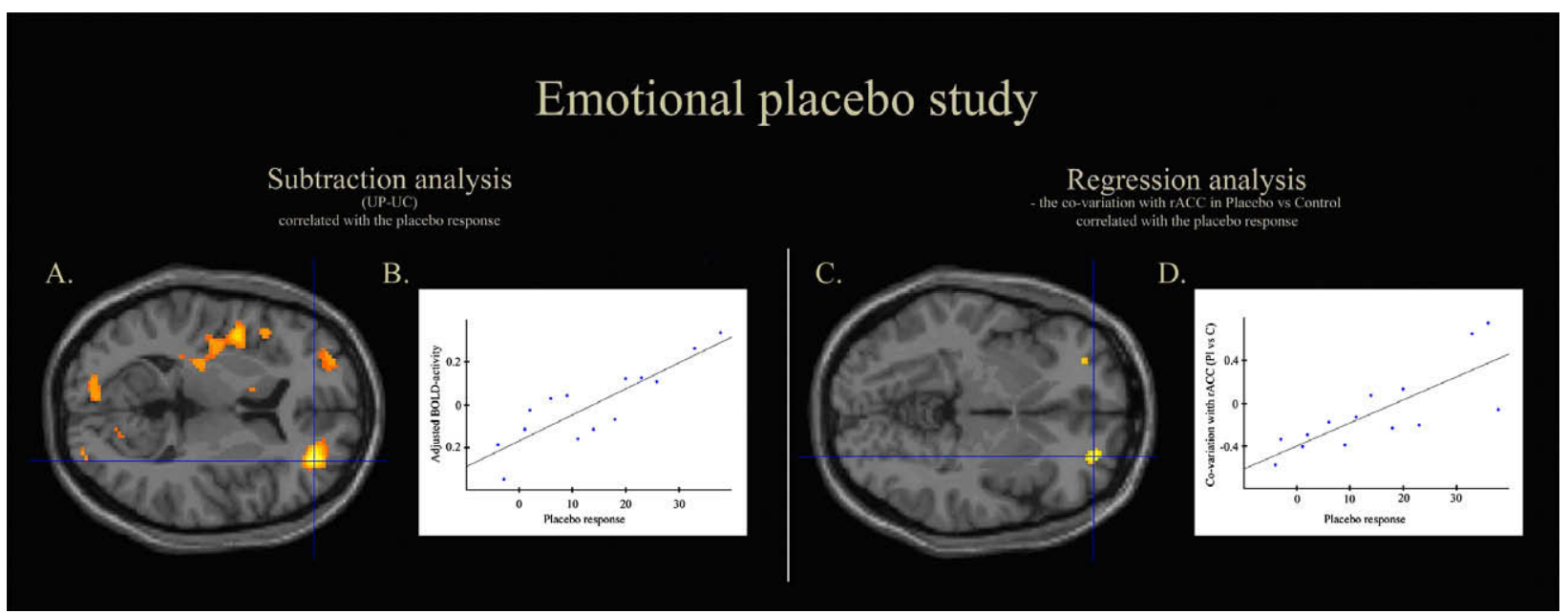

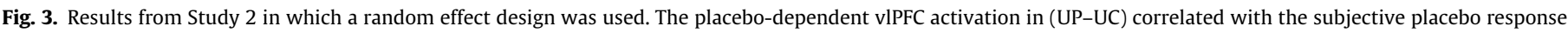

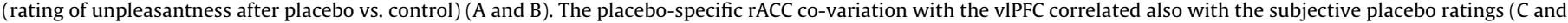
D).

Functional neuroimaging and neurophysiological studies suggest that the Obfc stores representations of the motivational value for specific primary and secondary re-enforcers [18,22,23,30,31, $45,46]$. The monitoring function involves representation of rewards and expectations of upcoming rewards $[30,31,41,45,46]$. Contemporary models of Obfc function suggest that one key function of this region is to signal the desirability of the expected outcomes and hold those associations on-line until the motivational goal has been achieved [42]. Moreover, prefrontal regions are important in generating prediction errors in associative learning [47] and also in pain processing [43].

Similar cognitive processes are involved in the placebo mechanism [14]. Explicit expectations of a relative reward (i.e. the expectation of pain relief) is a key function in the placebo analgesic response $[29,49,50]$, and treatment expectations correlate strongly with the subsequent placebo response [39]. We therefore suggest that the involvement of these prefrontal regions in placebo analgesia is related to the expectation of a treatment effect. A possible critique of this suggestion is that also the opioid analgesia condition should contain treatment expectation, and therefore activate these prefrontal regions to a similar degree. This is not challengeable in the anticipation phase. However, we propose that neither the expectation nor the error signals are the same during the two con-

\section{Table 4}

Study of the functional connectivity between rACC and right vlPFC/lObfc that correlated with the subjective placebo response (Study 2). In the present analysis random effect design was used.

\begin{tabular}{|c|c|c|c|c|c|}
\hline \multirow{2}{*}{$\begin{array}{l}\text { Region co-varying with rACC } \\
{[X Y Z]=\left[\begin{array}{lll}-12 & 48 & 12\end{array}\right]}\end{array}$} & \multicolumn{3}{|c|}{ MNI coordinates } & \multirow[t]{2}{*}{$t$-value } & \multirow[t]{2}{*}{$P$-value } \\
\hline & $X$ & $Y$ & $Z$ & & \\
\hline \multicolumn{6}{|l|}{ Placebo condition ( $r P C$ ) } \\
\hline Right vIPFC/lObfc & 28 & 54 & -4 & 3.36 & $0.003 \mathrm{~ns}$ \\
\hline Right vlPFC/lObfc & 34 & 54 & 8 & 2.73 & $0.009 \mathrm{~ns}$ \\
\hline Left $10 \mathrm{bfc} / \mathrm{lObfc}$ & -26 & 54 & -6 & 3.11 & $0.005 \mathrm{~ns}$ \\
\hline \multicolumn{6}{|l|}{ Control condition ( $\mathrm{rCC}$ ) } \\
\hline- & - & - & - & - & - \\
\hline \multicolumn{6}{|c|}{ Placebo condition vs. control condition (rPC-rCC) } \\
\hline Right vlPFC/lObfc & 28 & 52 & -4 & 4.36 & $<0.001$ \\
\hline Left vlPFC/lObfc & -26 & 50 & -6 & 3.25 & $0.003 \mathrm{~ns}$ \\
\hline Left lObfc/lObfc & -28 & 50 & -6 & 3.24 & $0.004 \mathrm{~ns}$ \\
\hline
\end{tabular}

rACC, rostral anterior cingulate cortex; lObfc, lateral orbitofrontal cortex; vlPFC ventrolateral prefrontal cortex; rCC, regression analysis during control condition; $\mathrm{rPC}$, regression analysis during placebo condition. ditions, since the opioid effect reduces the nociceptive input directly through opioid receptor activation while the placebo condition does not. In other words the subjects still expect a pain reduction during this condition, and will have a stronger error signal since the expectations do not match with the nociceptive input. This suggests that the vlPFC/Obfc may be more active in the placebo condition (due to stronger expectations and larger error signal) in line with our findings.

An important question is whether this prefrontal activity processing expectations and the error signal may also be involved in inducing the placebo response. It has been shown that expectations have a major impact on visual perceptual experience $[17,44,57]$ on affective experience [28], and elaborating upon those expectations requires the function of an intact Obfc $[9,12]$. Moreover, vlPFC and the lObfc have been implicated in top-down modulation of pain $[4,33,40]$ including placebo analgesia $[21,27,34,51]$, depression [13] and experimental anxiety [20,24,25,32]. These studies are in line with the idea that lateral ventro-orbital cortex is involved in a general, and not only pain specific, cognitive process that mediates modulation of pain and emotion [35].

A model for the mechanisms driving the placebo effect using expectations and error processing is that this is a consequence of Bayesian processing - as has been suggested for other perceptual systems $[16,17,57]$. In that hypothesis perceptions derived from external signals are a compromise between incoming signals and the expectations in the system. It has been suggested that this type of processing vastly increases efficiency of understanding the outer world.

While lObfc and vlPFC may be involved in cognitive processes driving the analgesic response during placebo, several receptor and functional imaging studies have not found the same type of an opioid-related involvement as shown for ACC and insula $[10,15,19,34,53,55,56]$. Here, we suggest that prefrontal regions may influence opioid systems in rACC - possibly through local enkephalinergic inter-neurons. It has been suggested that such inter-neurons may activate pyramidal projection neurons (through opioid receptors) that in turn may regulate distant pain-modulating areas [48]. In line with our hypothesis, our regression data showed the rACC co-varied with the lObfc/vlPFC in the placebo analgesia condition but not in the opioid analgesia condition. The same regions that co-varied with the rACC were also a part of the regions that were specifically activated during placebo treatment (PPL-POP) (Fig. 2B). We used the data from the study on 
emotional placebo [36] to probe whether this functional relation was directly related to the subjectively reported placebo response. In that study we have previously shown that both the rACC and the vlPFC correlate with the subjectively reported placebo effect and treatment expectations [36]. In the present analysis we show that the subjects who expressed the highest placebo response also had the most reliable placebo-specific coupling between the rACC and the vlPFC (Fig. 3C and D). Thus, we both replicated the placebo-specific functional relation between rACC and vlPFC and showed that it is directly related to the placebo effect. The functional connectivity analysis in the emotional placebo study generalizes the idea that prefrontal processes may interact with neuromodulatory systems in ACC to other areas than pain. Furthermore, the finding that activity in rACC may be opioid dependent also in emotional processing [37] suggests that the prefrontal cortex may drive opioidrelated modulatory systems in ACC during emotional placebo as well.

There are several alternative interpretations of the present results. Firstly, it cannot be excluded that a placebo-like increase in lObfc was masked in the opioid condition by pharmacologically induced decreases in CBF that is either neural or vascular in origin. Secondly, connectivity analysis does not show any causality - rACC may as well control prefrontal activity. It is also possible that the functional relation between rACC and lObfc represents a cognitive bias to report pain scores in line with one's prior beliefs. Moreover, the lack of rACC-Obfc correlation in the opioid condition may be explained by other factors than a fulfilled expectation, e.g. it would also be possible that a reduced level of consciousness or the heightened euphoric level during opioid condition attenuates this functional relation. Finally, we did not measure expectations although we used the reported decrease in unpleasantness during the conditioning day (day 1) as a proxy to treatment expectations in [36]. Interestingly, this value correlated to the placebo effect day 2 in both ACC and vlPFC. Finally, we only used open treatment in the opioid condition and it would be interesting to study the prefrontal involvement in open opioid treatment vs. hidden opioid treatment [11] associated with different degrees of treatment expectation.

In conclusion, the present results show that placebo analgesia is qualitatively different from an opioid drug response during pain. These findings and the suggestion that drugs directly interfere with the expectation pathway [11] indicate that a placebo response may not be interpreted as a passive control to a specific drug effect, but a highly active state in itself.

\section{Conflict of interest}

The authors of this manuscript have no financial or other relationship that may be perceived as a conflict of interest.

\section{Acknowledgments}

The research was supported by grants from the EFIC-Grünenthal Grant, Swedish Medical Research Council, the Karolinska Institute, BB/CBME, LA, FEDER/POCI 2010, the Knut and Alice Wallenberg Foundation, the Barbro and Bernard Osher Foundation, The Swedish Governmental Agency for Innovation Systems (VINNOVA), The Swedish Foundation for Strategic Research and the Petrus and Augusta Hedlunds Foundation. The authors are grateful to all participants from the MRC/PET facility and for all suggestions and comments from colleagues.

\section{Appendix A. Supplementary data}

Supplementary data associated with this article can be found, in the online version, at doi:10.1016/j.pain.2010.03.011.

\section{References}

[1] Adler LJ, Gyulai FE, Diehl DJ, Mintun MA, Winter PM, Firestone LL. Regional brain activity changes associated with fentanyl analgesia elucidated by positron emission tomography. Anesth Analg 1997;84:120-6.

[2] Amanzio M, Benedetti F. Neuropharmacological dissection of placebo analgesia: expectation-activated opioid systems versus conditioningactivated specific subsystems. J Neurosci 1999;19:484-94.

[3] Atweh SF, Kuhar MJ. Distribution and physiological significance of opioid receptors in the brain. Br Med Bull 1983;39:47-52.

[4] Bantick SJ, Wise RG, Ploghaus A, Clare S, Smith SM, Tracey I. Imaging how attention modulates pain in humans using functional MRI. Brain 2002;125: 310-9.

[5] Benedetti F. The opposite effects of the opiate antagonist naloxone and the cholecystokinin antagonist proglumide on placebo analgesia. Pain 1996;64: 535-43.

[6] Benedetti F, Amanzio M, Baldi S, Casadio C, Maggi G. Inducing placebo respiratory depressant responses in humans via opioid receptors. Eur J Neurosci 1999;11:625-31.

[7] Benedetti F, Arduino C, Amanzio M. Somatotopic activation of opioid systems by target-directed expectations of analgesia. J Neurosci 1999;19:3639-48.

[8] Bingel U, Lorenz J, Gläscher J, Weiller C, Büchel C. Mechanisms of placebo analgesia: rACC recruitment of a subcortical antinociceptive network. Pain 2006;120:8-15.

[9] Camille N, Coricelli G, Sallet J, Pradat-Diehl P, Duhamel J-R, Sirigu A. The involvement of orbitofrontal cortex in the experience of regret. Science 2004;304:1167-70.

[10] Casey KL, Svensson P, Morrow TJ, Raz J, Jone C, Minoshima S. Selective opiate modulation of nociceptive processing in the human brain. J Neurophysiol 2000;84:525-33.

[11] Colloca L, Benedetti F. Placebos and painkillers: is mind as real as matter? Nat Rev Neurosci 2005;6:545-52.

[12] Coricelli G, Critchley HD, Joffily M, O’Doherty J, Sirigu A, Dolan RJ. Regret and its avoidance: a neuroimaging study of choice behavior. Nat Neurosci 2005;8:1255-62.

[13] Drevets WC. Neuroimaging and neuropathological studies of depression: implications for the cognitive-emotional features of mood disorders. Curr Opin Neurobiol 2001;11:240-9.

[14] Fields H. State-dependent opioid control of pain. Nat Rev Neurosci 2005;5:565-75.

[15] Firestone LL, Gyulai F, Mintun M, Adler LJ, Urso K, Winter PM. Human brain activity response to fentanyl imaged by positron emission tomography. Anesth Analg 1996;82:1247-51.

[16] Friston K. A theory of cortical responses. Philos Trans R Soc Lond B Biol Sci 2005;360:815-36.

[17] Frith CD. Making up the mind: how the brain creates our mental world. Blackwell Publishing; 2007.

[18] Gottfried JA, O’Doherty J, Dolan RJ. Encoding predictive reward value in human amygdala and orbitofrontal cortex. Science 2003;301:1104-7.

[19] Jones AK, Qi LY, Fujirawa T, Luthra SK, Ashburner J, Bloomfield P, Cunningham VJ, Itoh M, Fukuda H, Jones T. In vivo distribution of opioid receptors in man in relation to the cortical projections of the medial and lateral pain systems measured with positron emission tomography. Neurosci Lett 1991;126: 25-8.

[20] Kalisch R, Wiech K, Critchley HD, Seymour B, O’Doherty JP, Oakley DA, Allen P, Dolan RJ. Anxiety reduction through detachment: subjective, physiological, and neural effects. J Cogn Neurosci 2005;17:874-83.

[21] Kong J, Gollub RL, Rosman IS, Webb JM, Vangel MG, Kirsch I, Kaptchuk TJ. Brain activity associated with expectancy-enhanced placebo analgesia as measured by functional magnetic resonance imaging. J Neurosci 2006;26:381-8.

[22] Kringelbach ML, Rolls ET. Neural correlates of rapid reversal learning in a simple model of human social interaction. Neurolmage 2003;20:1371-83.

[23] Kringelbach ML, Rolls ET. The functional neuroanatomy of the human orbitofrontal cortex: evidence from neuroimaging and neuropsychology. Prog Neurobiol 2004;72:341-72.

[24] Levesque J, Eugene F, Joanette Y, Paquette V, Mensour B, Beaudoin G, Leroux $J M$, Bourgouin P, Beauregard $M$. Neural circuitry underlying voluntary suppression of sadness. Biol Psychiatry 2003;53:502-10.

[25] Levesque J, Joanette Y, Mensour B, Beaudoin G, Leroux JM, Bourgouin P. Beauregard M. Neural basis of emotional self-regulation in childhood. Neuroscience 2004;129:361-9.

[26] Levine JD, Gordon NC, Fields HL. The mechanism of placebo analgesia. Lancet 1978;2:654-7.

[27] Lieberman MD, Jarcho JM, Berman S, Naliboff BD, Suyenobu BY, Mandelkern M, Mayer EA. The neural correlates of placebo effects: a disruption account. Neuroimage 2004;22:447-55.

[28] Mellers BA. Choice and the relative pleasure of consequences. Psychol Bull 2000;126:910-24.

[29] Montgomery GH, Kirsch I. Classical conditioning and the placebo effect. Pain 1997;72:107-13.

[30] O’Doherty J, Kringelbach ML, Rolls ET, Hornak J, Andrews C. Abstract reward and punishment representations in the human orbitofrontal cortex. Nat Neurosci 2001;4:95-102.

[31] O’Doherty JP, Deichmann R, Critchley HD, Dolan RJ. Neural responses during anticipation of a primary taste reward. Neuron 2002;33:815-26. 
[32] Ochsner KN, Bunge SA, Gross JJ, Gabrieli JD. Rethinking feelings: an FMRI study of the cognitive regulation of emotion. J Cogn Neurosci 2002;14: 1215-29.

[33] Petrovic P, Petersson KM, Ghatan PH, Stone-Elander S, Ingvar M. Pain-related cerebral activation is altered by a distracting cognitive task. Pain 2000;85: 19-30.

[34] Petrovic P, Kalso E, Petersson KM, Ingvar M. Placebo and opioid analgesia imagine a shared neuronal network. Science 2002;295:1737-40.

[35] Petrovic P. Opioid and placebo analgesia share the same network. Semin Pain Med 2005;3:31-6.

[36] Petrovic P, Dietrich T, Fransson P, Andersson J, Carlsson K, Ingvar M. Placebo in emotional processing-induced expectations of anxiety relief activate generalized modulatory network. Neuron 2005;46:957-69.

[37] Petrovic P, Pleger B, Seymour B, Kloppel S, De Martino B, Critchley H, Dolan RJ Blocking central opiate function modulates hedonic impact and anterior cingulate response to rewards and losses. J Neurosci 2008;28:10509-16.

[38] Pfeiffer A, Pasi A, Mehraein P, Herz A. Opiate receptor binding sites in human brain. Brain Res 1982;248:87-96.

[39] Price DD, Milling LS, Kirsch I, Duf FA, Montgomery GH, Nicholls SS. An analysis of factors that contribute to the magnitude of placebo analgesia in an experimental paradigm. Pain 1999;83:147-56.

[40] Rainville P, Hofbauer RK, Paus T, Duncan GH, Bushnell MC, Price DD. Cerebra mechanisms of hypnotic induction and suggestion. J Cogn Neurosci 1999;11: 110-25.

[41] Schoenbaum G, Chiba AA, Gallagher M. Orbitofrontal cortex and basolateral amygdala encode expected outcomes during learning. Nat Neurosci 1998;1: $155-9$.

[42] Schoenbaum G, Roesch M. Orbitofrontal cortex, associative learning, and expectancies. Neuron 2005;47:633-6.

[43] Seymour B, O’Doherty JP, Koltzenburg M, Wiech K, Frackowiak R, Friston K, Dolan R. Opponent appetitive-aversive neural processes underlie predictive learning of pain relief. Nat Neurosci 2005;8:1234-40.

[44] Sterzer P, Frith C, Petrovic P. Believing is seeing: expectations alter visual awareness. Curr Biol 2008;18:R697-8.

[45] Thorpe SJ, Rolls ET, Maddison S. The orbitofrontal cortex: neuronal activity in the behaving monkey. Exp Brain Res 1983;49:93-115.
[46] Tremblay L, Schultz W. Relative reward preference in primate orbitofrontal cortex [see comments]. Nature 1999;398:704-8.

[47] Turner DC, Aitken MRF, Shanks DR, Sahakian BJ, Robbins TW, Schwarzbauer C, Fletcher PC. The role of lateral frontal cortex in causal associative learning: exploring preventative and super-learning. Cereb Cortex 2004;14:872-80.

[48] Vogt BA, Finch DM, Olson CR. Functional heterogeneity in cingulate cortex: the anterior executive and posterior evaluative regions. Cereb Cortex 1992;2: 435-43.

[49] Voudouris NJ, Peck CL, Coleman G. Conditioned response models of placebo phenomena: further support. Pain 1989;38:109-16.

[50] Voudouris NJ, Peck CL, Coleman G. The role of conditioning and verbal expectancy in the placebo response. Pain 1990;43:121-8.

[51] Wager TD, Rilling JK, Smith EE, Sokolik A, Casey KL, Davidson RJ, Kosslyn SM, Rose RM, Cohen JD. Placebo-induced changes in FMRI in the anticipation and experience of pain. Science 2004;303:1162-7.

[52] Wager TD, Scott DJ, Zubieta JK. Placebo effects on human mu-opioid activity during pain. Proc Natl Acad Sci USA 2007;104:11056-61.

[53] Wagner KJ, Willoch F, Kochs EF, Siessmeier T, Tölle TR. Dose-dependent regional cerebral blood flow changes during remifentanil infusion in humans. A positron emission tomography study. Anesthesiology 2001;94:732-9.

[54] Wamsley JK, Zarbin MA, Young 3rd WS, Kuhar MJ. Distribution of opiate receptors in the monkey brain: an autoradiographic study. Neuroscience 1982;7:595-613.

[55] Willoch F, Tolle TR, Wester HJ, Munz F, Petzold A, Schwaiger M, Conrad B, Bartenstein $P$. Central pain after pontine infarction is associated with changes in opioid receptor binding: a PET study with ${ }^{11} \mathrm{C}$-diprenorphine. AJNR Am J Neuroradiol 1999;20:686-90.

[56] Willoch F, Schindler F, Wester HJ, Empl M, Straube A, Schwaiger M, Conrad B, Tolle TR. Central poststroke pain and reduced opioid receptor binding within pain processing circuitries: a $\left[{ }^{11} \mathrm{C}\right]$ diprenorphine PET study. Pain $2004 ; 108$ : 213-20.

[57] Yuille A, Kersten D. Vision as Bayesian inference: analysis by synthesis? Trends Cogn Sci 2006;10:301-8.

[58] Zubieta JK, Bueller JA, Jackson LR, Scott DJ, Xu Y, Koeppe RA, Nichols TE, Stohler CS. Placebo effects mediated by endogenous opioid activity on mu-opioid receptors. J Neurosci 2005;25:7754-62. 